Article

\title{
Functional Impact of the $N$-terminal Arm of Proline Dehydrogenase from Thermus thermophilus
}

\author{
Mieke M. E. Huijbers ${ }^{1}$, Ilona van Alen ${ }^{1}$, Jenny W. Wu ${ }^{1}$, Arjan Barendregt ${ }^{2,3}$, \\ Albert J. R. Heck ${ }^{2,3}$ (iD and Willem J. H. van Berkel ${ }^{1, *}$ \\ 1 Laboratory of Biochemistry, Wageningen University \& Research, Stippeneng 4, 6708 WE Wageningen, \\ The Netherlands; mieke.huijbers@wur.nl (M.M.E.H.); ilona.vanalen@wur.nl (I.v.A.); \\ jenny.wu@wur.nl (J.W.W.) \\ 2 Biomolecular Mass Spectrometry and Proteomics, Bijvoet Center for Biomolecular Research and Utrecht \\ Institute of Pharmaceutical Sciences, Utrecht University, Padualaan 8, 3584 Utrecht, The Netherlands; \\ a.barendregt@uu.nl (A.B.); a.j.r.heck@uu.nl (A.J.R.H.) \\ 3 Netherlands Proteomics Center, Padualaan 8, 3584 Utrecht, The Netherlands \\ * Correspondence: willem.vanberkel@wur.nl; Tel.: +31-6-120-77313
}

Received: 8 December 2017; Accepted: 14 January 2018; Published: 16 January 2018

\begin{abstract}
Proline dehydrogenase (ProDH) is a ubiquitous flavoenzyme that catalyzes the oxidation of proline to $\Delta^{1}$-pyrroline-5-carboxylate. Thermus thermophilus ProDH (TtProDH) contains in addition to its flavin-binding domain an $N$-terminal arm, consisting of helices $\alpha \mathrm{A}, \alpha \mathrm{B}$, and $\alpha \mathrm{C}$. Here, we report the biochemical properties of the helical arm truncated TtProDH variants $\triangle \mathrm{A}, \triangle \mathrm{AB}$, and $\triangle \mathrm{ABC}$, produced with maltose-binding protein as solubility tag. All three truncated variants show similar spectral properties as TtProDH, indicative of a conserved flavin-binding pocket. $\triangle \mathrm{A}$ and $\triangle \mathrm{AB}$ are highly active tetramers that rapidly react with the suicide inhibitor $\mathrm{N}$-propargylglycine. Removal of the entire $N$-terminal arm $(\triangle \mathrm{ABC})$ results in barely active dimers that are incapable of forming a flavin adduct with $N$-propargylglycine. Characterization of V32D, Y35F, and V36D variants of $\triangle \mathrm{AB}$ established that a hydrophobic patch between helix $\alpha \mathrm{C}$ and helix $\alpha 8$ is critical for TtProDH catalysis and tetramer stabilization.
\end{abstract}

Keywords: flavoprotein; proline dehydrogenase; protein engineering; protein oligomerization; solubility tag; suicide inhibition; TIM-barrel

\section{Introduction}

Proline dehydrogenase (ProDH; EC 1.5.5.2) is a ubiquitous enzyme involved in proline catabolism. ProDH catalyzes the flavin-dependent oxidation of L-proline to $\Delta^{1}$-pyrroline-5-carboxylate (P5C). After P5C hydrolysis, the resulting glutamic semialdehyde (GSA) is oxidized to glutamate through the action of $\Delta^{1}$-pyrroline-5-carboxylate dehydrogenase (P5CDH; EC 1.2.1.88) (Scheme 1). ProDH and $\mathrm{P} 5 \mathrm{CDH}$ exist as separate monofunctional enzymes in eukaryotes and some bacteria, but are fused into a bifunctional [1,2] or trifunctional [3] enzyme in other bacteria. In these multi-functional enzymes, called proline utilization A (PutA), the C-terminus of ProDH is fused to P5CDH, allowing for the channeling of the P5C/GSA intermediate between the enzymes [4-9].

ProDH has a distorted $(\beta \alpha)_{8}$ TIM-barrel fold (Figure 1A), which is conserved throughout the PutA/ProDH family [11,12]. Opposed to the classic TIM-barrel fold, the ProDH barrel begins with a helix $(\alpha 0)$ rather than a strand (Figure 1). This extra helix occupies the location that is normally reserved for $\alpha 8$. As a consequence, $\alpha 8$ is not located alongside $\beta 8$, but on top of the barrel $[1,13,14]$. The distorted location of $\alpha 8$ is crucial for catalysis, since it contributes three strictly conserved residues (Tyr-x-x-Arg-Arg) that interact with the substrate [13,14]. 


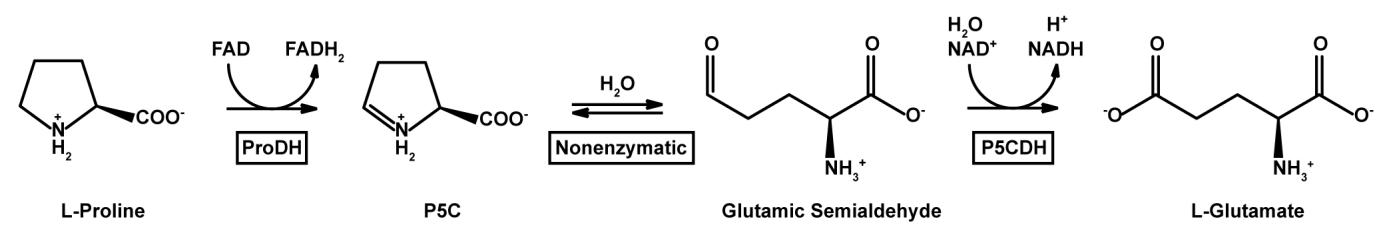

Scheme 1. Conversion of L-proline to L-glutamate by proline dehydrogenase (ProDH) and $\Delta^{1}$-pyrroline-5-carboxylate dehydrogenase (P5CDH). Scheme based on [10].

The $N$-terminal sequence of ProDH is poorly conserved. Monofunctional eukaryotic ProDHs, including the human enzyme [15], have an elongated $N$-terminus when compared to monofunctional bacterial ProDHs [11,12]. ProDH from Thermus thermophilus (TtProDH) contains an $N$-terminal arm consisting of three helices: $\alpha \mathrm{A}, \alpha \mathrm{B}$, and $\alpha \mathrm{C}$ (Figure 1). Helix $\alpha 8$ fits into the cleft, which is formed by helices $\alpha \mathrm{A}, \alpha \mathrm{B}$ and $\alpha \mathrm{C}$. This cleft is assumed to be involved in channeling P5C/GSA between TtProDH and TtP5CDH $[1,16]$.

Previously, we showed that TtProDH is overproduced in Escherichia coli (E. coli) when its $\mathrm{N}$-terminus is fused to maltose-binding protein (MBP) [17] and that the recombinant enzyme does not discriminate between FAD and FMN as cofactor [18]. Replacing Phe10 and Leu12 in helix $\alpha$ A with Glu residues (further referred to as MBP-TtProDH variant EE) successfully released MBP-TtProDH from non-native self-association, yielding homogeneous tetramers [19]. Although TtProDH crystallizes as a dimer (Figure 1) [1], it is worthy of note that in solution, the enzyme remains a tetramer after removal of the MBP solubility tag [13].

A

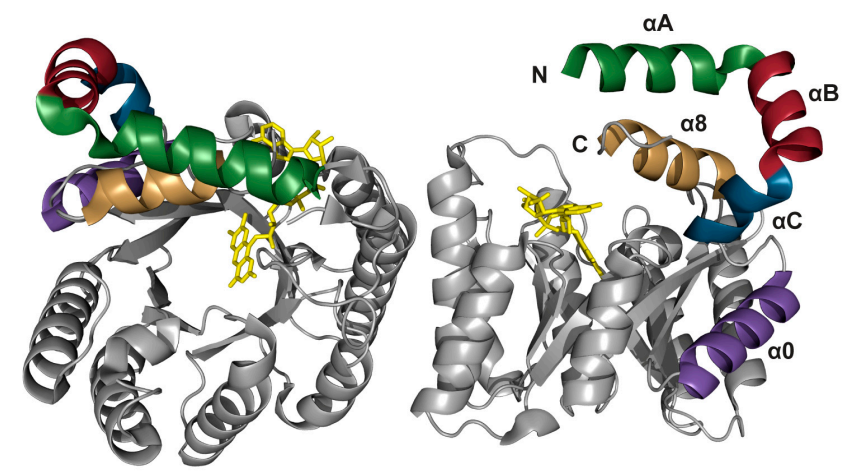

B

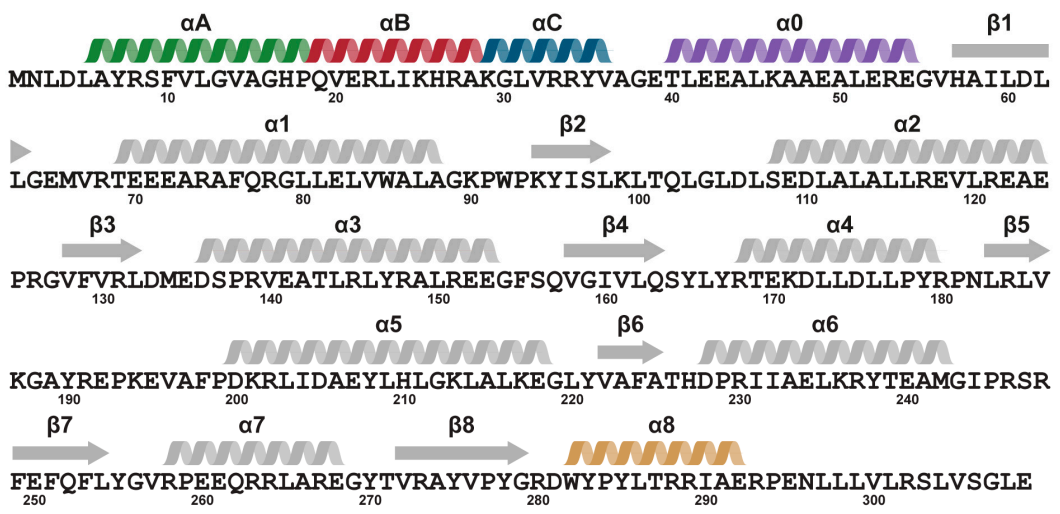

Figure 1. Structural features of Thermus thermophilus proline dehydrogenase (TtProDH). (A) Three-dimensional model of the crystal structure of the TtProDH dimer (PDB entry 2G37). The $N$-terminal helices $\alpha \mathrm{A}$ (green), $\alpha \mathrm{B}$ (red), and $\alpha \mathrm{C}$ (blue) are indicated, as well as helix $\alpha 0$ (purple) and the C-terminal helix $\alpha 8$ (brown); (B) Amino acid sequence of TtProDH. Secondary structural elements on top of the sequence have the same colors as in Figure 1A. Figure 1A is based on [19]. 
To investigate the functional impact of the $N$-terminal arm of TtProDH in catalysis and oligomerization in closer detail, we constructed MBP-fused variants, lacking, respectively, one $(\Delta \mathrm{A})$, two $(\triangle \mathrm{AB})$, or three $(\triangle \mathrm{ABC}) N$-terminal helices. $\triangle \mathrm{A}$ and $\triangle \mathrm{AB}$ turned out to be highly active tetramers, while $\triangle \mathrm{ABC}$ was an almost inactive dimer. To further probe the role of helix $\alpha \mathrm{C}$, we addressed the properties of site-specific variants V32D, Y35F, and V36D of $\triangle \mathrm{AB}$. This revealed that a hydrophobic patch between helix $\alpha \mathrm{C}$ and helix $\alpha 8$ is critical for TtProDH catalysis and tetramer stabilization.

\section{Results}

\subsection{Protein Expression and Purification}

MBP-TtProDH EE, $\triangle \mathrm{A}, \triangle \mathrm{AB}$ and $\triangle \mathrm{ABC}$ were overproduced in E. coli TOP10 cells. From $1 \mathrm{~L}$ of culture about 200-250 mg of each variant was purified, yields that we have described before for the heterologous production of MBP-TtProDH [17,19]. From SDS-PAGE analysis of the purified enzymes, it can be appreciated that sequential removal of three helices results in a gradual decrease of subunit molecular mass (Figure 2).

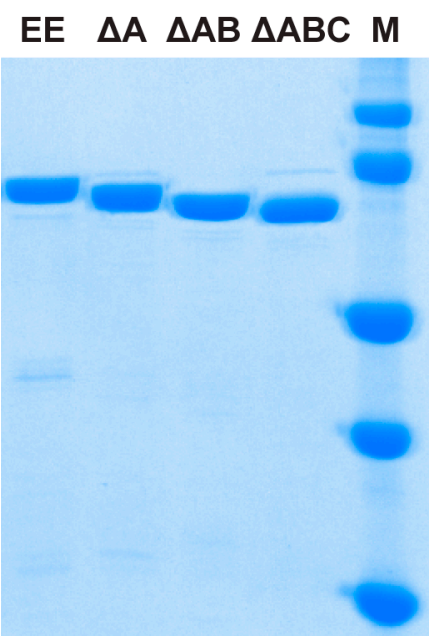

Figure 2. Purified MBP-TtProDH variants visualized on sodium dodecyl sulfate polyacrylamide gel electrophoresis (SDS-PAGE). Molecular masses of marker proteins (M), from top to bottom: 100, 75, 50, 37, $25 \mathrm{kDa}$.

Previously, we demonstrated that removal of the MBP fusion tag with trypsin does not significantly affect the catalytic properties and oligomerization behavior of TtProDH [17]. However, as found with EE [19], trypsinolysis of the arm-truncated variants did not result in homogeneous preparations. Therefore, we used the MBP-fused variants for further studies.

\subsection{Spectral Properties}

The far-UV CD spectra of EE and the $N$-terminal variants are very similar (Figure 3A) and comparable to that of MBP-TtProDH [17]. The visible flavin absorption spectra of EE and the arm-truncated variants (Figure 3B) are also nearly identical to that of MBP-TtProDH [17,19], except that the lowest energy absorption band of $\triangle \mathrm{ABC}$ has shifted about $2 \mathrm{~nm}$ to higher wavelengths. These data support that there are no major structural changes and that deletion of the $N$-terminal helices does not significantly alter the microenvironment of the flavin-binding site. A recent crystallographic study confirmed that $\triangle \mathrm{ABC}$ (residues 38-279; PDB 5M42) has a similar overall structure as TtProDH (PDB 2G37) with an rmsd value of $0.338 \AA$ (for $221 \mathrm{C} \alpha$ atoms of A chains) $[1,18]$. 

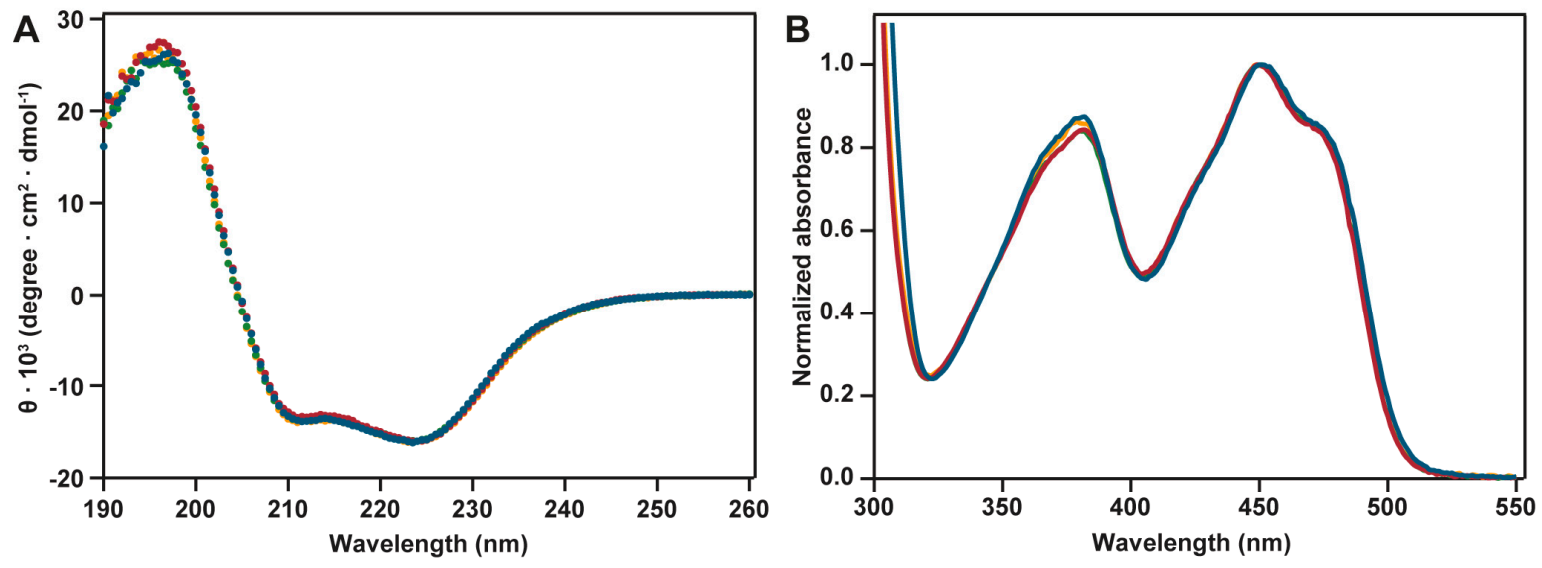

$-\mathrm{EE}-\Delta \mathrm{A}-\Delta \mathrm{AB}-\Delta \mathrm{ABC}$

Figure 3. Spectral properties of EE, $\triangle \mathrm{A}, \triangle \mathrm{AB}$, and $\triangle \mathrm{ABC}$. (A) Far-UV CD spectra; (B) Visible flavin absorption spectra.

\subsection{Hydrodynamic Properties}

EE purifies as a tetramer $[17,19]$. The truncated variants $\triangle \mathrm{A}$ and $\triangle \mathrm{AB}$ also form tetramers as indicated by size exclusion chromatography (Figure $4 \mathrm{~A}$ ). $\triangle \mathrm{ABC}$ elutes mainly as a dimer (Figure $4 \mathrm{~A}$ ), suggesting that $\alpha \mathrm{C}$ plays a role in the tetramerization process. Previously, we showed that TtProDH prepared from MBP-TtProDH also forms tetramers, but that the addition of $1 \mathrm{M} \mathrm{GuHCl}$ induces the formation of dimers [17]. The crystal structure also shows a dimeric structure (cf. Figure 1A).
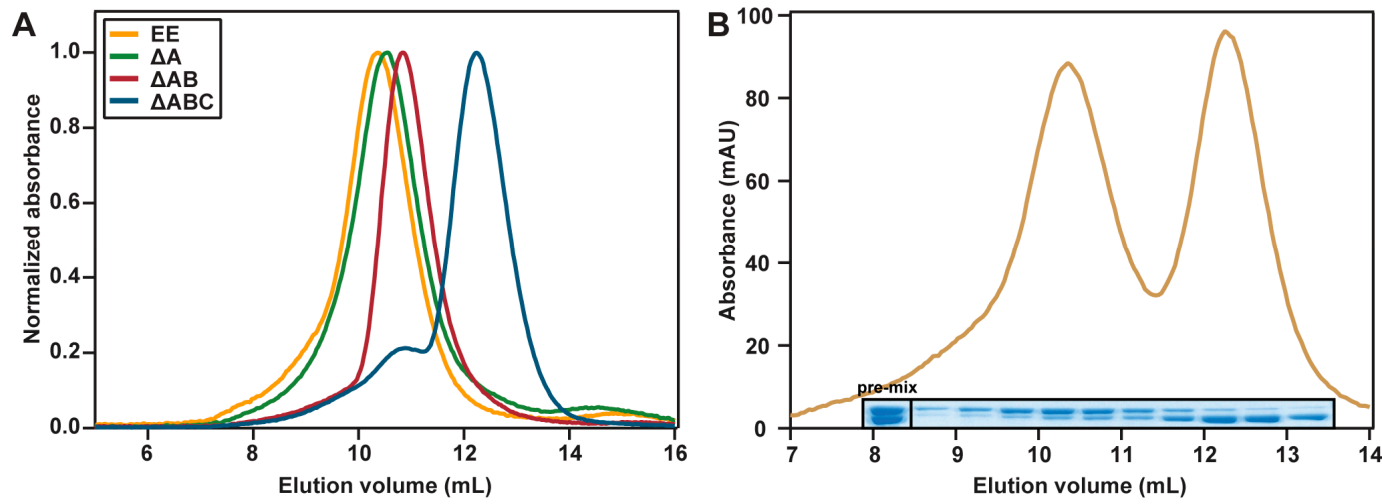

Figure 4. Hydrodynamic properties of $\mathrm{EE}, \triangle \mathrm{A}, \triangle \mathrm{AB}$, and $\triangle \mathrm{ABC}$ as monitored by Superdex 200 size-exclusion chromatography. (A) Elution pattern of the MBP-TtProDH variants; (B) Hydrodynamic properties of $\mathrm{EE}$ and $\triangle \mathrm{ABC}$, mixed in equal amounts and equilibrated at room temperature overnight. SDS-PAGE of the gel filtration fractions is also shown.

Mixing of EE and $\triangle \mathrm{ABC}$ followed by analytical gel filtration reveals two separate peaks, a peak for the tetrameric species of $E E$ and a peak for the dimeric species of $\triangle A B C$ (Figure $4 B$ ). This indicates that mixing of the two enzyme forms does not lead to EE- $\triangle \mathrm{ABC}$ association. Moreover, SDS-PAGE of the gel filtration fractions shows that no subunit exchange occurs (inset Figure 4B).

Additional information about the oligomeric state of the different variants was obtained by native mass spectrometry. The estimated masses (Table 1 ) confirm that $\triangle \mathrm{A}$ and $\triangle \mathrm{AB}$ exist predominantly as tetramers, and $\triangle \mathrm{ABC}$ as dimer. With $\triangle \mathrm{ABC}$, minor amounts of tetramers are present, in agreement with the analytical gel filtration results (Figure $4 \mathrm{~A}$ ). For $\triangle \mathrm{A}$ and $\triangle \mathrm{AB}$, some dimers are observed with native mass spectrometry, and for $\triangle \mathrm{ABC}$ monomers, although their abundance is rather low. 
Denaturing the different complexes enabled an accurate mass measurement of the individual subunits (Table 1). These masses show that all of the variants exist of identical subunits.

Table 1. Molecular masses of EE, $\triangle \mathrm{A}, \triangle \mathrm{AB}$, and $\triangle \mathrm{ABC}$ as determined by native and denatured ESI-MS. Both predicted (Pred.) as well as experimental (Exp.) masses are given in $\mathrm{kDa}$ with experimental errors less than $0.01 \%$. For calculation of the predicted native masses, it has been taken into account that each subunit contains a non-covalently bound FAD cofactor (molecular mass $786 \mathrm{Da}$ ).

\begin{tabular}{|c|c|c|c|c|c|c|c|c|}
\hline & \multicolumn{6}{|c|}{ Native } & \multicolumn{2}{|c|}{ Denatured } \\
\hline & \multicolumn{2}{|c|}{ Tetramer } & \multicolumn{2}{|c|}{ Dimer } & \multicolumn{2}{|c|}{ Monomer } & \multirow[b]{2}{*}{ Pred. } & \multirow[b]{2}{*}{ Exp } \\
\hline & Pred. & Exp. & Pred. & Exp. & Pred. & Exp. & & \\
\hline MBP-TtProDH EE * & 317.8 & 319.6 & 158.9 & 158.9 & 79.5 & - & 78.7 & 78.7 \\
\hline MBP-TtProDH $\Delta A$ & 310.1 & 311.3 & 155.0 & $154.9-155.7$ & 77.5 & - & 76.7 & 76.8 \\
\hline MBP-TtProDH $\triangle \mathrm{AB}$ & 305.1 & $307.6-307.8$ & 152.6 & $152.6-153.7$ & 76.3 & - & 75.5 & 75.5 \\
\hline MBP-TtProDH $\triangle \mathrm{ABC}$ & 300.7 & $290.0-290.7$ & 150.4 & $145.0-146.0$ & 75.2 & $71.9-72.7$ & 74.4 & 71.9 \\
\hline MBP-TtProDH $\triangle \mathrm{AB}$ V32D & 305.2 & $296.2-304.4$ & 152.6 & $146.5-149.0$ & 76.3 & $73.0-76.0$ & 75.5 & $\begin{array}{l}73.0 \\
75.5\end{array}$ \\
\hline MBP-TtProDH $\triangle \mathrm{AB}$ Y35F & 305.1 & 307.4 & 152.5 & $152.3-152.9$ & 76.3 & - & 75.5 & 75.5 \\
\hline MBP-TtProDH $\triangle \mathrm{AB}$ V36D & 305.2 & 296.2 & 152.6 & $151.1-151.9$ & 76.3 & - & 75.5 & $\begin{array}{l}73.0 \\
75.5\end{array}$ \\
\hline
\end{tabular}

* As determined previously [19].

The experimental masses of native and denatured $\triangle \mathrm{ABC}$ do not correspond with the predicted masses. The observed species appears to be C-terminally truncated [18]. Based on the estimated mass, cleavage occurs before Arg288, leading to the removal of a part of the C-terminal tail with sequence (288-RRIAERPENLLLVLRSLVSGLE-309). This truncated form has a predicted subunit mass of 71,884.9 Da, close to the measured mass of 71,899.3 Da [18].

\subsection{Catalytic Properties}

Figure 5 presents an overview of the steady-state kinetic properties of the MBP-TtProDH variants. The kinetic parameters derived from these experiments are summarized in Table 2 . The proline $K_{\mathrm{m}}$ values of $\mathrm{EE}, \triangle \mathrm{A}, \triangle \mathrm{AB}$, and $\triangle \mathrm{ABC}$ are comparable. However, $\triangle \mathrm{A}$ and $\triangle \mathrm{AB}$ have a slightly higher activity than $\mathrm{EE}$ while $\triangle \mathrm{ABC}$ is almost inactive. These data suggest that $\alpha \mathrm{A}$ and $\alpha \mathrm{B}$ are not required for optimal activity, and that further deletion of helix $\alpha \mathrm{C}$ is critical for catalysis.

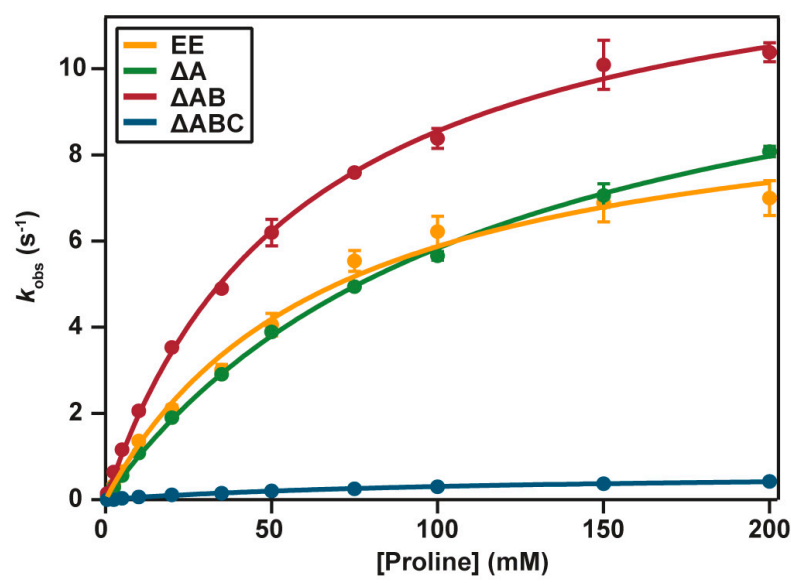

Figure 5. Steady-state kinetics of $\mathrm{EE}, \triangle \mathrm{A}, \triangle \mathrm{AB}$, and $\triangle \mathrm{ABC}$. The fitted curves are retrieved from non-linear regression analysis applying the Michaelis-Menten equation. Each data point was retrieved in triplicate. 
Table 2. Kinetic parameters of the MBP-TtProDH variants at $25^{\circ} \mathrm{C}, \mathrm{pH} 7.4$, as determined with the Proline:DCPIP oxidoreductase assay, using proline as the variable substrate, and specific activities of the MBP-TtProDH variants as determined by the proline: $\mathrm{O}_{2}$ assay, at a concentration of $100 \mathrm{mM}$ proline. Each data point for the Proline:DCPIP assay was retrieved in triplicate; the data points for the Proline: $\mathrm{O}_{2}$ assay were retrieved in duplicate. The raw kinetic data of the Proline:DCPIP assay, used to determine the $K_{\mathrm{m}}$ and $k_{\text {cat }}$, is provided in Table $\mathrm{S} 1$.

\begin{tabular}{|c|c|c|c|c|}
\hline & \multicolumn{3}{|c|}{ Proline:DCPIP Assay } & \multirow{2}{*}{$\begin{array}{c}\text { Proline: } \mathrm{O}_{2} \text { Assay } \\
\text { Specific Activity (mU/mg) }\end{array}$} \\
\hline & $K_{\mathrm{m}}(\mathrm{mM})$ & $k_{\text {cat }}\left(\mathrm{s}^{-1}\right)$ & $k_{\mathrm{cat}} / K_{\mathrm{m}}\left(\mathrm{s}^{-1} \mathrm{M}^{-1}\right)$ & \\
\hline MBP-TtProDH EE * & $68 \pm 8$ & $9.8 \pm 0.5$ & 146 & $266 \pm 12$ \\
\hline MBP-TtProDH $\Delta \mathrm{A}$ & $116 \pm 5$ & $12.6 \pm 0.3$ & 109 & $405 \pm 15$ \\
\hline MBP-TtProDH $\triangle \mathrm{AB}$ & $60 \pm 3$ & $13.6 \pm 0.3$ & 229 & $228 \pm 8$ \\
\hline MBP-TtProDH $\triangle \mathrm{ABC}$ & $114 \pm 8$ & $0.7 \pm 0.02$ & 6 & $20 \pm 1$ \\
\hline MBP-TtProDH $\triangle \mathrm{AB}$ V32D & $309 \pm 25$ & $3.2 \pm 0.2$ & 10 & $265 \pm 4$ \\
\hline MBP-TtProDH $\triangle \mathrm{AB}$ Y35F & $161 \pm 29$ & $14.1 \pm 1.5$ & 88 & $388 \pm 5$ \\
\hline MBP-TtProDH $\Delta$ AB V36D & $189 \pm 27$ & $0.3 \pm 0.03$ & 1.6 & $21 \pm 1$ \\
\hline
\end{tabular}

* As determined previously [19].

Since TtProDH has a low but significant proline oxidase activity [1], it was of interest to address the oxygen reactivity of the $\mathrm{N}$-terminal arm variants. For all of the variants described above, micromolar concentrations of enzyme were needed to reliable measure consumption of oxygen, and very low specific activities were observed (Table 2). Nevertheless, these data confirm that removal of the complete $N$-terminal arm $(\triangle \mathrm{ABC})$ also impairs the proline oxidase activity of MBP-TtProDH.

\subsection{Reaction with N-propargylglycine}

TtProDH is irreversibly inactivated by the suicide inhibitor $N$-propargylglycine [10]. Inactivation involves the initial oxidation of $N$-propargylglycine to $N$-propargyliminoglycine and the subsequent formation of a bicovalent linkage between flavin $\mathrm{N}(5)$ and the $\varepsilon$-amino group of Lys99 (Figure 6C). Lys99 is located in the loop between $\beta 2$ and $\alpha 2$ and is involved in binding the carboxylic moiety of proline [1]. Upon reaction with $N$-propargylglycine the absorption maximum of TtProDH at $450 \mathrm{~nm}$ disappears, while the maximum around $380 \mathrm{~nm}$ gradually increases. Furthermore, the peak at $380 \mathrm{~nm}$ shifts to longer wavelengths. This behavior is indicative of the initial reduction of the flavin and the subsequent formation of the covalent Lys99-FAD adduct $[8,10]$. After reaction with $N$-propargylglycine, the enzyme is locked in the reduced state.

To probe the catalytic features of the MBP-TtProDH variants in further detail, we investigated the reactivity of $\mathrm{EE}$ and the arm-truncated variants with $\mathrm{N}$-propargylglycine. Figure 6A shows that all of the variants except $\triangle \mathrm{ABC}$ form a covalent flavin adduct, and that the reactions result in similar absorption changes, as observed before with TtProDH [10]. However, a more careful analysis of the kinetics of the reactions reveals significant differences.

For $\mathrm{EE}, \Delta \mathrm{A}$, and $\triangle \mathrm{AB}$, flavin reduction and adduct formation are clearly observed (Figure $6 \mathrm{~A}$ ). Reduction of EE by $N$-propargylglycine is relatively slow, as evidenced from the time-dependent decrease in absorption at $450 \mathrm{~nm}$. Flavin reduction is immediately followed by covalent adduct formation as evidenced from the absorbance increase around 380 and $405 \mathrm{~nm}$. Variants $\triangle \mathrm{A}$ and $\triangle \mathrm{AB}$ reveal an increased rate of reduction compared to EE. This corresponds with the increased $k_{\text {cat }}$ values for these variants (Table 2). The peak at $380 \mathrm{~nm}$ shows a red shift to $385 \mathrm{~nm}$ for EE and to $388 \mathrm{~nm}$ for $\triangle \mathrm{A}$ and $\triangle \mathrm{AB}$. Furthermore, both for $\triangle \mathrm{A}$ and $\triangle \mathrm{AB}$, the increase in absorbance around $388 \mathrm{~nm}$ is more pronounced.

$\triangle \mathrm{ABC}$ shows neither flavin reduction nor formation of a flavin adduct (Figure 6A). However, a slow rise in absorption with a maximum at $290 \mathrm{~nm}$ is observed in the near-UV region, pointing to the conversion of $\mathrm{N}$-propargylglycine to $\mathrm{N}$-propargyliminoglycine. Activity measurements with DCPIP suggest that $N$-propargylglycine is indeed a poor substrate for $\triangle \mathrm{ABC}$. 
Activity of the different enzyme variants before and after incubation with $2.5 \mathrm{mM}$ of $\mathrm{N}$-propargylglycine was measured (Figure 6B). For EE, residual activity after 90 min of incubation with the inhibitor is about $13 \%$. For $\triangle \mathrm{A}$ and $\triangle \mathrm{AB}$ the activity loss is even more pronounced, in good agreement with the spectral results. The residual activity of $\triangle \mathrm{ABC}$ after $90 \mathrm{~min}$ incubation with $\mathrm{N}$-propargylglycine is extremely low (Figure 6B).

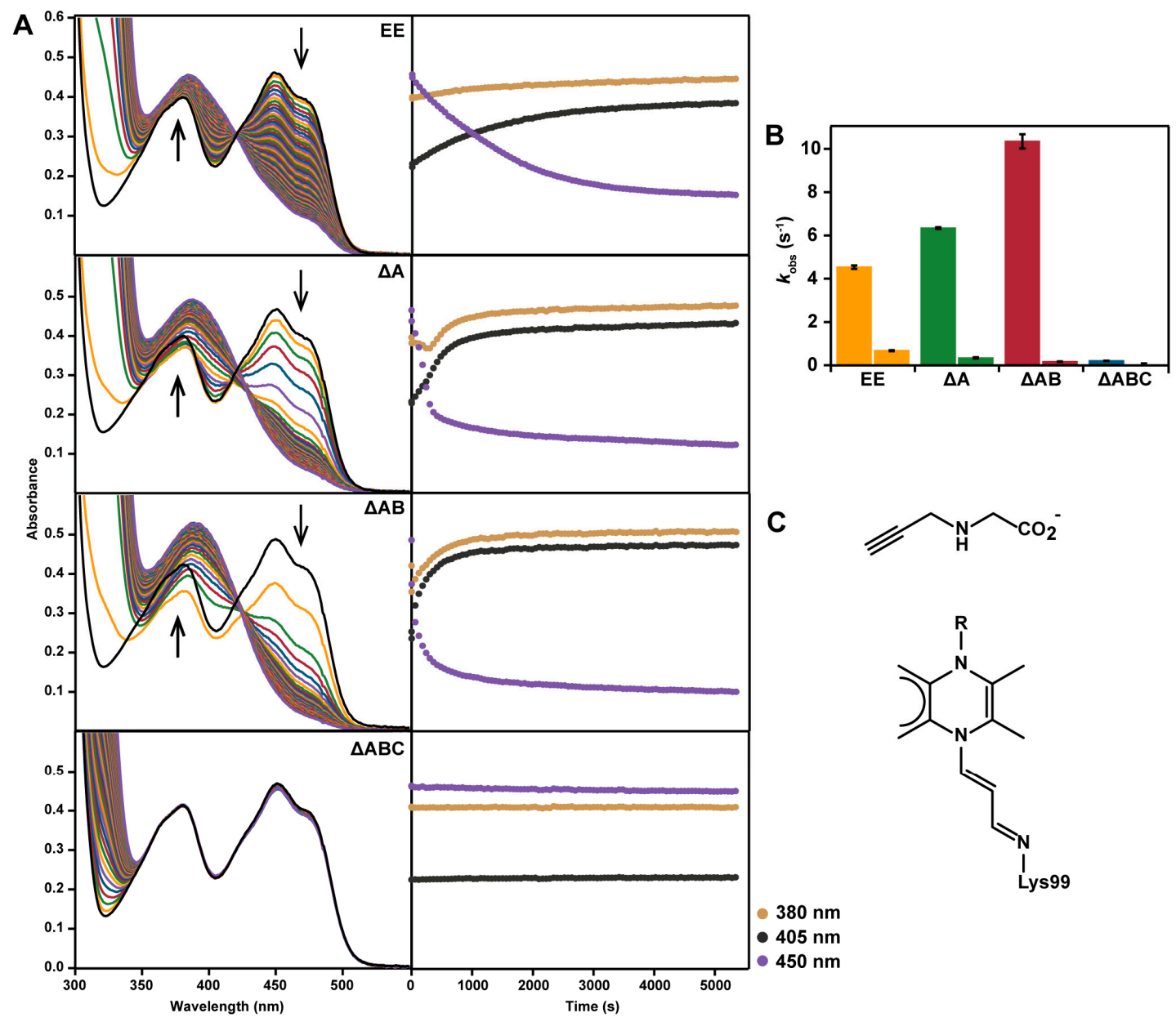

Figure 6. Reactivities of the $N$-terminal arm TtProDH variants with $N$-propargylglycine. (A) Left: absorption spectral changes of the variants upon incubation with $N$-propargylglycine. The black line indicates the spectrum before the addition of $N$-propargylglycine. Spectra were recorded at $1 \mathrm{~min}$ intervals for $90 \mathrm{~min}$. Right: absorbance changes of the variants upon incubation with $\mathrm{N}$-propargylglycine followed at 380, 405, and $450 \mathrm{~nm}$; (B) Activity of the different enzyme variants before and after incubation with $N$-propargylglycine. Error bars are based on three replicates.

(C) Chemical structure of $\mathrm{N}$-propargylglycine and the covalent Lys99-FAD adduct.

\subsection{Interactions between Helix $\alpha$ C and Helix $\alpha 8$}

$\triangle \mathrm{ABC}$ is produced as a catalytically impaired dimer. In addition, this MBP-TtProDH variant is truncated at its $C$-terminal helix $\alpha 8$. This suggests that helix $\alpha \mathrm{C}$ is important for the stabilization of helix $\alpha 8$. The three-dimensional model of the crystal structure of TtProDH suggests that there is a hydrogen bond between Tyr35 of $\alpha \mathrm{C}$ and Glu295 of $\alpha 8$ (Figure 7). Glu295 is part of the sequence that is cleaved off in $\triangle \mathrm{ABC}$. To investigate whether the Tyr-Glu interaction is important for the stabilization of helix $\alpha 8$, we changed Tyr35 to Phe. In addition, there is a hydrophobic patch between helix $\alpha \mathrm{C}$ and $\alpha 8$ (Figure 7). To examine the importance of this patch for the stabilization of helix $\alpha 8$, Val32, and Val36 individually were changed into Asp. The single amino acid substitutions were introduced in 
MBP-TtProDH $\triangle \mathrm{AB}$, since this variant is fully active and forms stable tetramers. In this way, we only look at the interaction between helix $\alpha \mathrm{C}$ and $\alpha 8$ without possible interference of helix $\alpha \mathrm{A}$ and $\alpha \mathrm{B}$.

A

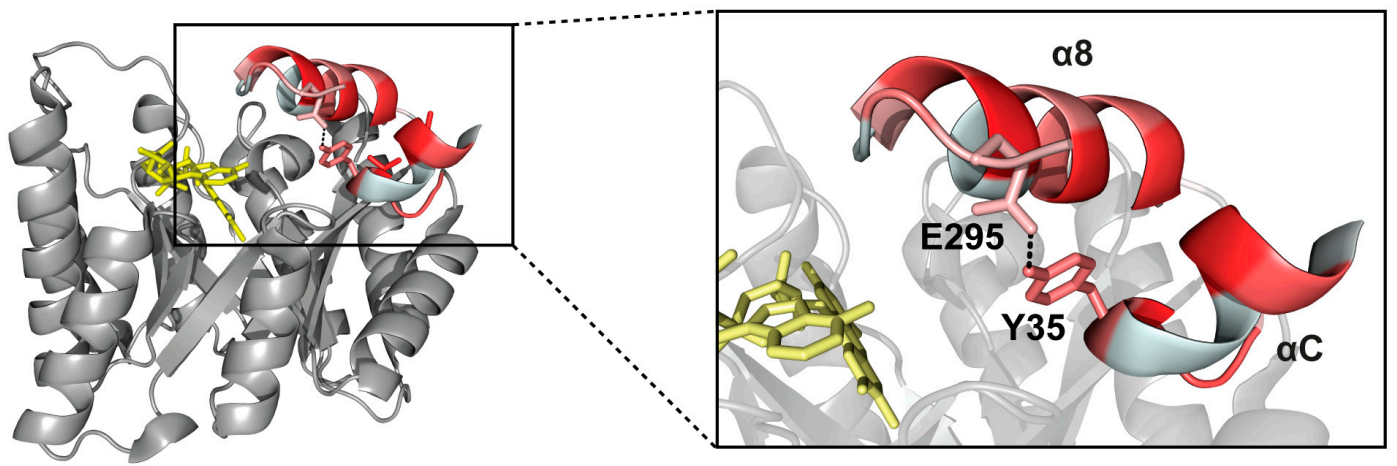

B

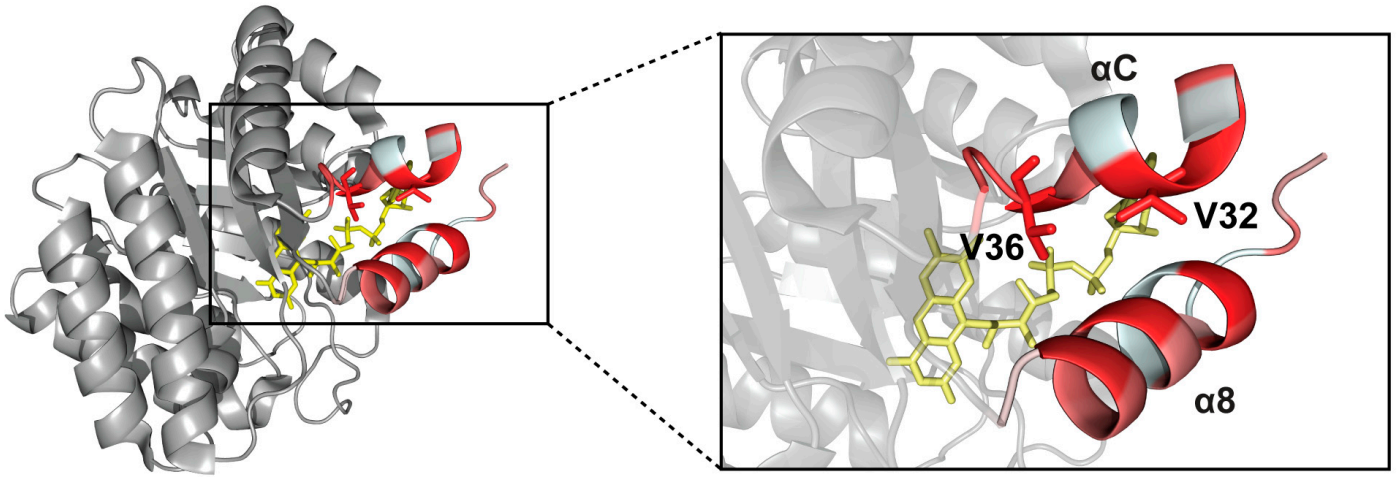

Figure 7. Interactions between helix $\alpha \mathrm{C}$ and helix $\alpha 8$. The residues in helices $\alpha \mathrm{C}$ and $\alpha 8$ are colored in a hydrophobic (red) to non-hydrophobic (white) gradient, according to the hydrophobicity scale introduced by Eisenberg et al. [20]. The remaining part of the catalytic domain is shown in grey and the FAD cofactor is shown in yellow. (A) Ion pair Tyr35 $(\alpha$ C) and Glu295 $(\alpha 8)$; (B) Hydrophobic patch between $\alpha \mathrm{C}$ and $\alpha 8$ with Val32 $(\alpha \mathrm{C})$ and Val36 $(\alpha \mathrm{C})$ indicated. Data according to the crystal structure of TtProDH (PDB entry 2G37).

The far-UV CD spectra of V32D, Y35F, and V36D (Figure 8A) are identical to the far-UV CD-spectra of the $\mathrm{N}$-terminal variants (Figure $3 \mathrm{~A}$ ). The visible flavin absorption properties of the $\triangle \mathrm{AB}$ variants show that the low energy absorption bands of V32D and V36D have shifted about $2 \mathrm{~nm}$ to higher wavelength, as compared to those of $\triangle \mathrm{AB}$ and $\mathrm{Y} 35 \mathrm{~F}$ (Figures $3 \mathrm{~B}$ and $8 \mathrm{~B}$ ). Thus, the flavin absorption properties of V32D and V36D resemble those of $\triangle \mathrm{ABC}$ (Figure $3 \mathrm{~B}$ ).

Size exclusion chromatography of Y35F indicates that substitution of Tyr35 with Phe does not affect the tetrameric nature of $\triangle \mathrm{AB}$ (Figure 8C). Therefore, the interaction between Tyr35 and Glu295 does not seem critical for stabilization of helix $\alpha 8$. V32D and V36D elute much later from the gel filtration column than Y35F, suggesting that these variants mainly form dimers (Figure 8C). Native MS of $\mathrm{Y} 35 \mathrm{~F}$ confirms the presence of tetramers. Furthermore, V32D and V36D are present both as tetramers and dimers (Table 1). The mass of denatured Y35F shows the presence of identical subunits, while V32D and V36D show two subunit masses, of which one corresponds with the predicted mass (Table 1). The differences between the subunit masses suggest that both V32D and V36D are partially intact and partially cleaved before R288 (predicted mass 73,001.2 Da), indicating flexibility and instability of helix $\alpha 8$. 

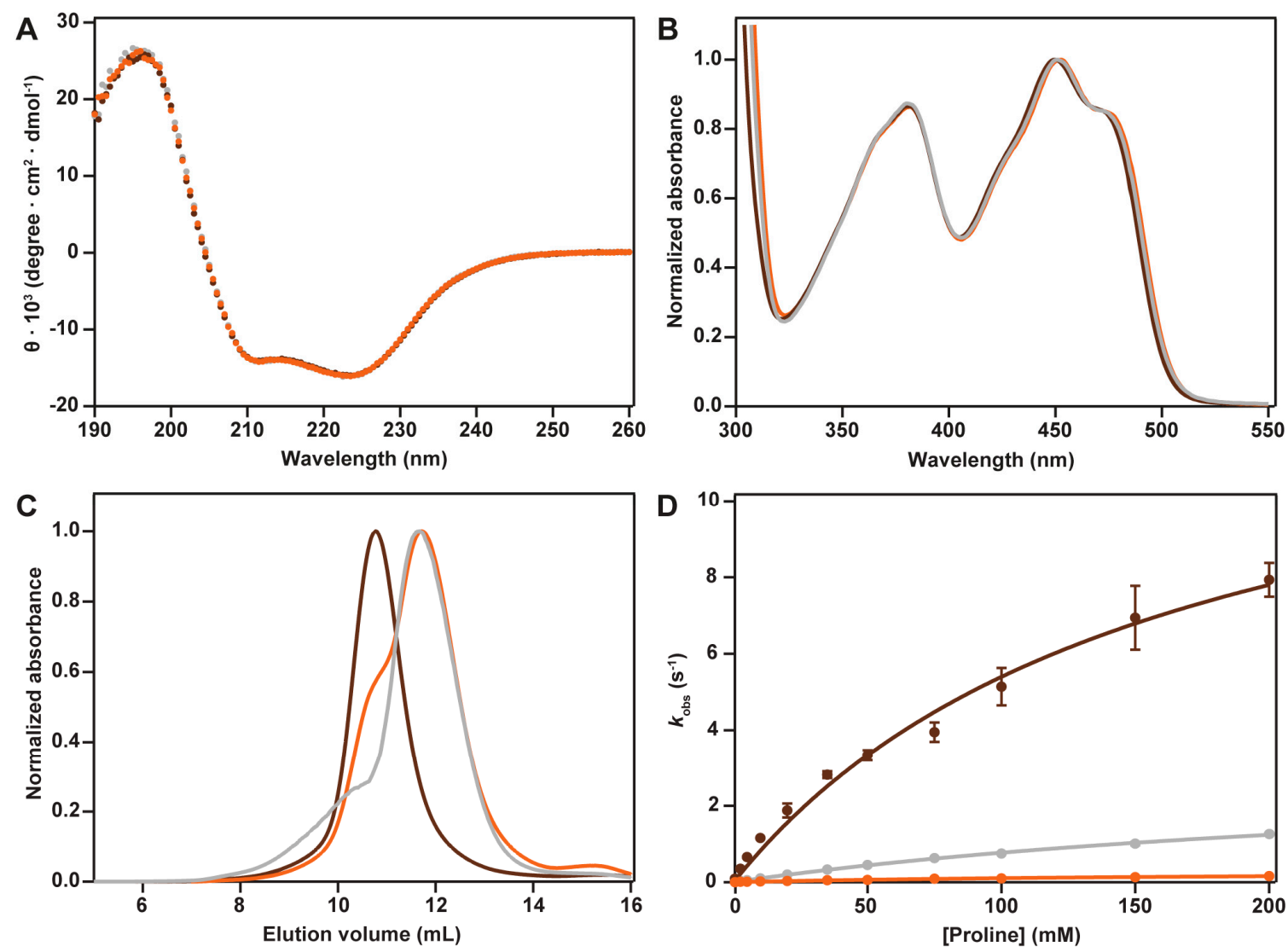

Figure 8. Properties of helix $\alpha \mathrm{C}$ variants of $\triangle \mathrm{AB}$. (A) Far-UV CD spectra; (B) Visible flavin absorption spectra; (C) Hydrodynamic properties as monitored by Superdex 200 size-exclusion chromatography; and, (D) Steady-state kinetic data as determined with the Proline:DCPIP assay. Each data point was retrieved in triplicate. The raw kinetic data of the Proline:DCPIP assay, used to determine the $K_{\mathrm{m}}$ and $k_{\text {cat }}$ values, is provided in Table S1.

Analysis of the catalytic properties of Y35F confirms that the interaction between Tyr35 and Glu295 is not crucial for the functioning of TtProDH (Figure 8D). The minor decrease in catalytic efficiency when compared to $\triangle \mathrm{AB}$ mainly results from a slight increase in $K_{\mathrm{m}}$ for the proline substrate (Table 2). V32D and V36D, on the other hand, are poorly active (Figure 8D). With these variants, a considerable decrease in catalytic efficiency is observed (Table 2). Oxidase activity of especially V36D is very low (Table 2), as found with $\triangle \mathrm{ABC}$.

The catalytic features of the helix $\alpha \mathrm{C}$ variants were investigated in more detail by probing their reactivity with the suicide inhibitor $\mathrm{N}$-propargylglycine (Figure 9). For Y35F (Figure 9A), the rates for flavin reduction and adduct formation are similar as found for $\triangle \mathrm{AB}$ (Figure $6 \mathrm{~A}$ ). For V32D, flavin reduction and adduct formation are also observed (Figure 9A), but these processes are much slower than for $\triangle \mathrm{AB}$. The flavin prosthetic group of $\mathrm{V} 36 \mathrm{D}$ is not reduced by $\mathrm{N}$-propargylglycine, and the typical absorption increase around $380 \mathrm{~nm}$, indicative for adduct formation, is also not observed (Figure 9A). However, as for $\triangle A B C$, a slow rise in absorption occurs in the region 300-350 nm, pointing to the conversion of $\mathrm{N}$-propargylglycine to $\mathrm{N}$-propargyliminoglycine.

All the helix $\alpha \mathrm{C}$ variants lose activity when treated with $N$-propargylglycine. After incubation for $90 \mathrm{~min}, \mathrm{Y} 35 \mathrm{~F}$ is almost completely inactivated, but V32D and V36D retain 30\% and $63 \%$ of their original (rather low) activity (Figure 9B). 


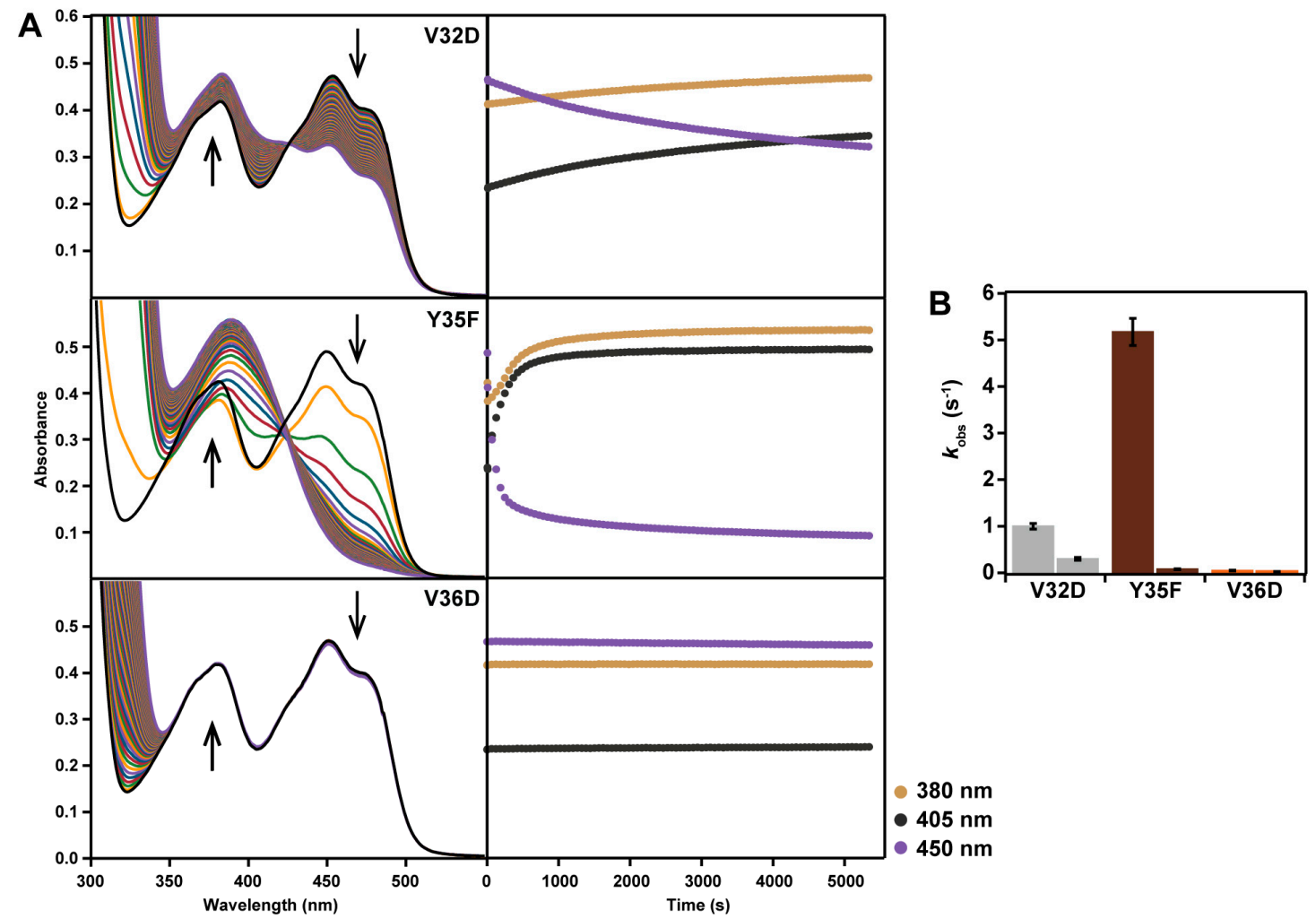

Figure 9. Reactivities of helix $\alpha \mathrm{C}$ variants of $\triangle \mathrm{AB}$ with $N$-propargylglycine. (A) Left: absorption spectral changes of the variants upon incubation with $N$-propargylglycine. The black line indicates the spectrum before the addition of $N$-propargylglycine. Spectra were recorded at 1 min intervals for 90 min. Right: absorbance changes of the variants upon incubation with $N$-propargylglycine followed at 380,405, and $450 \mathrm{~nm}$; (B) Activity of the different enzyme variants before and after incubation with $\mathrm{N}$-propargylglycine. Error bars are based on three replicates.

\section{Discussion}

Proline dehydrogenases contain a conserved $(\beta \alpha)_{8}$ TIM-barrel domain and an $N$-terminal arm that differs in length among monofunctional bacterial and eukaryotic ProDHs. In this study, we investigated the functional impact of the $N$-terminal arm of Thermus thermophilus ProDH. We analyzed variants that lack one $(\triangle \mathrm{A})$, two $(\triangle \mathrm{AB})$, or three $(\triangle \mathrm{ABC}) N$-terminal helices and compared these variants to the $\mathrm{EE}$ variant of MBP-fused TtProDH. The latter variant is a highly active soluble form of the enzyme that exclusively forms tetramers [19]. Spectral analysis showed that truncation of the $N$-terminal arm of $\mathrm{TtProDH}$ does neither affect the binding of the FAD cofactor nor the microenvironment of the flavin isoalloxazine ring.

We already showed that non-native aggregation of MBP-TtProDH is due to the hydrophobicity of helix $\alpha$ A. Replacing Phe10 and Leu12 of $\alpha$ A with glutamates eliminates the formation of larger aggregates [19]. Here, we established that the complete removal of helix $\alpha \mathrm{A}(\triangle \mathrm{A})$, or removal of helices $\alpha \mathrm{A}$ and $\alpha \mathrm{B}(\triangle \mathrm{AB})$, has the same effect: no aggregates are observed and $\Delta \mathrm{A}$ and $\triangle \mathrm{AB}$ exclusively form tetramers. This confirms that $\alpha \mathrm{A}$ is responsible for the in vitro aggregation of MBP-TtProDH and that both helices $\alpha \mathrm{A}$ and $\alpha \mathrm{B}$ are not essential for the tetramerization process. Furthermore, estimation of kinetic parameters revealed that $\alpha \mathrm{A}$ and $\alpha \mathrm{B}$ are not essential for the enzymatic activity of MBP-TtProDH. Actually, $\triangle \mathrm{AB}$ is the most active MBP-fused TtProDH variant reported thus far. Incubations with the mechanism-based inhibitor $N$-propargylglycine yielded supporting information about the catalytic competence and the structural integrity of $\triangle \mathrm{A}$ and $\triangle \mathrm{AB}$. The flavin cofactor of 
both arm-truncated variants is rapidly reduced, immediately followed by covalent adduct formation. This leads to irreversible suicide inactivation of the enzymes by $\mathrm{N}$-propargylglycine (Figure 6).

Helix $\alpha \mathrm{C}$ turned out to be more crucial for oligomerization and catalysis. Removal of the complete $N$-terminal arm $(\triangle \mathrm{ABC})$ resulted in catalytically impaired dimers and poor reactivity with $N$-propargylglycine. MS analysis showed that $\triangle \mathrm{ABC}$ has lost 22 residues at the $C$-terminus, including Arg288 and Arg289, involved in proline binding [1]. Thus, the dissociation of tetramers into dimers and loss in catalytic performance of $\triangle \mathrm{ABC}$ might be caused by the removal of $\alpha \mathrm{C}$ and/or the partial absence of helix $\alpha 8$. We do not have indications that the loss in activity is caused by dimer formation, since the dimer-dimer interactions in the tetramer seem to be relatively weak. The oxidase activity remains low for all deletion variants, suggesting that the removal of the $N$-terminal helices does not improve the access of oxygen to the flavin.

For Deinococcus radiodurans ProDH (DrProDH, PDB 4H6Q) [21], Bradyrhizobium japonicum PutA (BjPutA, PDB 3HAZ) [22] and Geobacter sulfurreducens PutA (GsPutA, PDB 4NM9) [7], a conserved Arg-Glu ion pair (Arg288-Glu65 in TtProDH) (Figure S1) is suggested to act as an active site gate. Arg288 is present in helix $\alpha 8$ while Glu65 is present in the $\beta 1-\alpha 1$ loop. In the presence of substrate, this loop moves towards the active site and the Arg-Glu ion pair can form. In $\triangle A B C$, helix $\alpha 8$ is cleaved before Arg288, thereby disrupting this ion pair and excluding this possibility for active site stabilization.

Triggered by the properties of $\triangle \mathrm{ABC}$, we introduced single amino acid substitutions in $\triangle \mathrm{AB}$ that might disrupt the interaction between helix $\alpha \mathrm{C}$ and helix $\alpha 8$ in a more delicate way. Replacement of Tyr35 with Phe showed that the hydrogen bond interaction between Tyr35 and Glu259 is not crucial for the catalytic performance of the enzyme, and also not for protein tetramerization. Incubation with $\mathrm{N}$-propargylglycine confirmed that the flavin reactivity and structural integrity of Y35F are highly comparable to that of $\triangle \mathrm{AB}$.

The Tyr35-Glu295 interaction is not conserved in DrProDH, although both amino acid residues are present in a region with highly conserved sequence. The latter enzyme is the only other monofunctional ProDH of which a crystal structure is available [21]. In DrProDH, Tyr35 is replaced by a phenylalanine (Phe34), while Glu295 is replaced by an arginine (Arg298). The absence of the Tyr-Glu interaction in DrProDH is another indication that this ion pair is not of great importance for the structural integrity of TtProDH.

The V32D and V36D variants of $\triangle \mathrm{AB}$ provided further insight into the functional role of helix $\alpha \mathrm{C}$. Both of the variants mainly form dimers, show partial proteolytic processing at the $C$-terminus, and display low catalytic efficiencies. This lends support to the proposal that the hydrophobic patch between helix $\alpha \mathrm{C}$ and $\alpha 8$ (Figure 7) is not only important for tetramer formation, but also for the proper functioning of the active site. Assessment of the reactivity of V32D and V36D with $\mathrm{N}$-propargylglycine revealed interesting differences. While slow flavin reduction and covalent adduct formation are observed for V32D, these processes do not take place with V36D. In agreement with this, V36D is less active with proline than V32D, and resembles $\triangle \mathrm{ABC}$ in this respect (Table 2). When we compare the hydrophobicity of helices $\alpha \mathrm{C}$ and $\alpha 8$ against their counterparts in $\mathrm{DrProDH}$, we observe a similar hydrophobic patch between both helices. In addition, analysis of the crystal structures of E. coli PutA (PDB 4O8A), BjPutA, and GsPutA suggests that helix $\alpha 8$ might also be stabilized by hydrophobic contacts, although in PutA enzymes helix $\alpha 8$ might also be stabilized by additional $N$ and $C$-terminal helices.

In conclusion, our results strengthen the idea that helix $\alpha \mathrm{C}$ is involved in TtProDH tetramerization and stabilization of helix $\alpha 8$. The hydrophobic patch between helix $\alpha \mathrm{C}$ and helix $\alpha 8$ stimulates tetramerization and is important for catalysis because it orients helix $\alpha 8$ for proper binding of the substrate and interaction with the active site.

We have shown that helix $\alpha \mathrm{A}$ and $\alpha \mathrm{B}$ of TtProDH are not crucial for the in vitro activity of the enzyme. However, $\alpha \mathrm{A}$ and $\alpha \mathrm{B}$ of TtProDH might be of importance in vivo, by serving as a docking interface for partner enzyme TtP5CDH during channeling [16] and/or for interaction with the 
membrane. Concerning these issues, it is of interest to study the function of the elongated $N$-terminus of monofunctional eukaryotic ProDHs, especially of human ProDH [15].

\section{Materials and Methods}

\subsection{Construction of MBP-TtProDH Variants}

Three $N$-terminally shortened MBP-fused variants were constructed; each variant was shortened with one additional $N$-terminal helix. To amplify the DNA, pBAD-MBP-TtProDH [19] was used as template DNA. The primers listed in Table 3 were used for amplification. Using EcoRI and HindIII restriction sites, the amplified fragments were introduced into a pBAD-MBP vector, which resulted in $N$-terminal fusions of the TtProDH variants to MBP. The resulting constructs MBP-TtProDH $\triangle \mathrm{A}$, MBP-TtProDH $\triangle \mathrm{AB}$, and MBP-TtProDH $\triangle \mathrm{ABC}$ were verified by automated sequencing of both strands (Macrogen, Seoul, Korea) and the plasmids were transformed to E. coli TOP10 host cells for recombinant expression.

Table 3. Oligonucleotides used for the construction of the various MBP-TtProDH variants. For the helix $\alpha \mathrm{C}$ variants, codon changes are underlined.

\begin{tabular}{|c|c|}
\hline Variant & Oligonucleotide Sequence ( $5^{\prime}$ to $\left.3^{\prime}\right)$ \\
\hline$\Delta \mathrm{A}$, forward & AATTAGAATTCCAGGTTGAACGTCTGATTAAACATCGTGCAAAAGG \\
\hline$\Delta \mathrm{AB}$, forward & AAT TAGAATTCAAAGGTCTGGTTCGTCGTTATGTTGCCGGTG \\
\hline$\triangle \mathrm{ABC}$, forward & AATTAGAATTCCAGGTTGAACGTCTGATTAAACATCGTGCAAAAGG \\
\hline$\Delta \mathrm{A}, \triangle \mathrm{AB}, \triangle \mathrm{ABC}$, reverse & GCCCAAGCTTTTATTCTAGACCGCTAACCAGGC \\
\hline$\triangle \mathrm{AB}, \mathrm{V} 32 \mathrm{D}$, forward * & CGAGGGAAGGATTTCAGAATTCAAAGGTCTGGATCGTCGTTATGTTGCCGGTGAAACCCTGG \\
\hline$\triangle \mathrm{AB}, \mathrm{Y} 35 \mathrm{~F}$, forward * & CGAGGGAAGGATTTCAGAATTCAAAGGTCTGGTTCGTCGTTTTGTTGCCGGTGAAACCCTGG \\
\hline$\triangle \mathrm{AB}, \mathrm{V} 36 \mathrm{D}$, forward * & CGAGGGAAGGATTTCAGAATTCAAAGGTCTGGTTCGTCGTTATGATGCCGGTGAAACCCTGG \\
\hline
\end{tabular}

The obtained plasmid for MBP-TtProDH $\triangle \mathrm{AB}$ was used as a template to construct point mutations in helix $\alpha \mathrm{C}$. V32D, Y35F, and V36D were constructed using the procedure described earlier [19], with the exception that in this case both a forward and a reverse primer were used (Table 3 ).

\subsection{Expression and Purification of MBP-TtProDH Variants}

The MBP-TtProDH variants were purified following a previously described procedure [19]. In short, the variants were produced in E. coli TOP10 cells and purified using amylose affinity and anion-exchange chromatography.

\subsection{Protein Analysis}

Enzyme purity was checked with sodium dodecyl sulfate polyacrylamide gel electrophoresis (SDS-PAGE). 10\% polyacrylamide slab gels were used and the proteins were stained with Coomassie Brilliant Blue R-250. $0.5 \mu \mathrm{g}$ of purified $N$-terminal variants was loaded per lane. As a molecular weight marker, Precision Plus Protein Standard (Biorad, Hercules, CA, USA) was used.

\subsection{Analytical Gel Filtration}

The hydrodynamic properties of the MBP-TtProDH variants were analyzed by size exclusion chromatography, as described previously [19]. In addition, $20 \mu \mathrm{M} E \mathrm{E}$ and $20 \mu \mathrm{M} \triangle \mathrm{ABC}$ in $50 \mathrm{mM}$ sodium phosphate $\mathrm{pH} 7.4$ were mixed, incubated at room temperature overnight, and subsequently analyzed by size exclusion chromatography. 


\subsection{ESI-MS}

The native and denatured masses of the MBP-TtProDH variants were determined using nanoflow electrospray ionization mass spectrometry (ESI-MS) according to a previously established procedure $[17,19]$, whereby the settings were optimized for the current application. Source backing pressure was increased to 7.8 mbar and the cone voltage was varied between 100 and $150 \mathrm{~V}$.

\subsection{Spectral Analysis}

Far-UV circular dichroism (CD) spectra of the MBP-TtProDH variants were recorded, as described before [17]. $1 \mu \mathrm{M}$ samples were prepared in $50 \mathrm{mM}$ sodium phosphate, $\mathrm{pH}$ 7.4. Optical flavin absorption spectra of the MBP-TtProDH variants were recorded as has been described previously [19].

\subsection{Enzyme Activity}

Enzyme activity of the MBP-TtProDH variants was determined at $25^{\circ} \mathrm{C}$ on a Hewlett Packard 8453 diode array spectrophotometer (Agilent Technologies, Santa Clara, CA, USA) using the proline:dichlorophenolindophenol (DCPIP) oxidoreductase assay [17]. For the standard assay, $0.07-0.66$ of enzyme was added to a $600 \mu \mathrm{L}$ reaction mixture containing $65 \mu \mathrm{M}$ DCPIP and $100 \mathrm{mM}$ L-proline in $50 \mathrm{mM}$ sodium phosphate $\mathrm{pH}$ 7.4. Steady-state kinetic parameters were determined at $25^{\circ} \mathrm{C}$, essentially as described previously [19].

Proline oxidase activity of the MBP-TtProDH variants was determined at $25{ }^{\circ} \mathrm{C}$ in air-saturated $50 \mathrm{mM}$ sodium phosphate $\mathrm{pH} 7.4$, containing 3.5-7.5 $\mu \mathrm{M}$ enzyme, using a Hansatech Oxytherm system (Hansatech Instruments, King's Lynn, UK). Reactions were started by the addition of $100 \mathrm{mM}$ L-proline.

\subsection{Inactivation with $N$-propargylglycine}

The synthesis of $\mathrm{N}$-propargylglycine was based on a procedure described before [23]. A clear solution of iodoacetic acid $(1.05 \mathrm{~g}, 5.6 \mathrm{mmol})$ and $\mathrm{N}$-propargylamine $(2.6 \mathrm{~g}, 47 \mathrm{mmol})$ was refluxed in $50 \mathrm{~mL}$ aqueous ethanol for $24 \mathrm{~h}$. The dark mixture was cooled to room temperature and the solvent was removed in vacuo. The crude product was precipitated from 1:1 ethanol:ethyl acetate. Recrystallization from aqueous ethanol and ethyl acetate and final drying under high vacuum yielded the $N$-propargylglycine as a white solid $(28 \mathrm{mg}, 4 \%) .{ }^{1} \mathrm{H}-\mathrm{NMR}\left(\mathrm{DMSO}_{6}\right): \delta=3.42(\mathrm{~d}, 2 \mathrm{H}, J=2.4 \mathrm{~Hz})$, $3.24(\mathrm{~s}, 2 \mathrm{H}), 3.16(\mathrm{t}, 1 \mathrm{H}, J=2.4 \mathrm{~Hz})$.

Spectral changes that were associated with the reaction of TtProDH with $N$-propargylglycine were monitored at $25^{\circ} \mathrm{C}$ on a Hewlett Packard 8453 diode array spectrophotometer. A fresh stock solution of $75 \mathrm{mM} \mathrm{N}$-propargylglycine was prepared in $50 \mathrm{mM}$ sodium phosphate $\mathrm{pH} 7.4 .40 \mu \mathrm{M}$ of the MBP-TtProDH variants in $50 \mathrm{mM}$ sodium phosphate $\mathrm{pH} 7.4$ was incubated with a final concentration of $2.5 \mathrm{mM} \mathrm{N}$-propargylglycine. Immediately after the addition of $\mathrm{N}$-propargylglycine, the first spectrum was recorded. Subsequent spectra were recorded at $1 \mathrm{~min}$ intervals for $90 \mathrm{~min}$. Before and after the incubation of the enzyme variants with $N$-propargylglycine, $10 \mu \mathrm{L}$ aliquots were removed from the cuvette and the enzyme activity was determined with the standard assay. Activity measurements were performed in triplicate.

Supplementary Materials: The following are available, Table S1: Raw kinetic data of the Proline:DCPIP assay of $\mathrm{EE}, \triangle \mathrm{A}, \triangle \mathrm{AB}, \triangle \mathrm{ABC}$ and helix $\alpha \mathrm{C}$ variants V32D, Y35F and V36D. Figure S1: Alignment of TtProDH, DrProDH, and the ProDH domains of BjPutA and GsPutA.

Acknowledgments: We thank Adrie Westphal (Laboratory of Biochemistry, Wageningen University) for technical assistance. We are indebted to Sebastiaan A. van den Berg (Laboratory of Organic Chemistry, Wageningen University) for the synthesis of $\mathrm{N}$-propargylglycine. MH received financial support from the Netherlands Organization for Scientific Research (NWO) and The Graduate School VLAG (Wageningen, The Netherlands) through the ERA-NET Industrial Biotechnology program (ERA-IB-2, project EIB.10.004) of the European Community. The mass spectrometry research was performed within the framework of the Netherlands Organisation for Scientific Research (NWO) and supported by the large-scale proteomics facility Proteins@Work (project 184.032.201) embedded in The Netherlands Proteomics Centre. 
Author Contributions: W.J.H.v.B. supervised the study. M.M.E.H. and W.J.H.v.B. designed the experiments. M.M.E.H., I.v.A. and J.W.W. performed the experiments. M.M.E.H. analyzed the data. A.B. and A.J.R.H. were responsible for the mass spectrometry data. M.M.E.H. and W.J.H.v.B. wrote the manuscript.

Conflicts of Interest: The authors declare no conflict of interest.

\section{Abbreviations}

$\begin{array}{ll}\text { CD } & \text { circular dichroism } \\ \text { DCPIP } & \text { dichlorophenolindophenol } \\ \text { ESI-MS } & \text { electron spray ionization mass spectrometry } \\ \text { FAD } & \text { flavin adenine dinucleotide } \\ \text { FMN } & \text { flavin mononucleotide } \\ \text { GSA } & \text { glutamic semialdehyde } \\ \text { MBP } & \text { maltose-binding protein } \\ \text { ProDH } & \text { proline dehydrogenase } \\ \text { P5C } & \Delta^{1} \text {-pyrroline-5-carboxylate } \\ \text { P5CDH } & \Delta^{1} \text {-pyrroline-5-carboxylate dehydrogenase } \\ \text { PutA } & \text { proline utilization A } \\ \text { SDS-PAGE } & \text { sodium dodecyl sulfate polyacrylamide gel electrophoresis } \\ \text { Tt } & \text { Thermus thermophilus } \\ \text { EE } & \text { MBP-TtProDH with Phe10 and Leu12 replaced by Glu } \\ \Delta \mathrm{A} & \text { MBP-TtProDH lacking helix } \alpha \mathrm{A} \\ \Delta \mathrm{AB} & \text { MBP-TtProDH lacking helices } \alpha \mathrm{A} \text { and } \alpha \mathrm{B} \\ \Delta \mathrm{ABC} & \text { MBP-TtProDH lacking helices } \alpha \mathrm{A}, \alpha \mathrm{B} \text { and } \alpha \mathrm{C}\end{array}$

\section{References}

1. White, T.A.; Krishnan, N.; Becker, D.F.; Tanner, J.J. Structure and kinetics of monofunctional proline dehydrogenase from Thermus thermophilus. J. Biol. Chem. 2007, 282, 14316-14327. [CrossRef] [PubMed]

2. Korasick, D.A.; Gamage, T.T.; Christgen, S.; Stiers, K.M.; Beamer, L.J.; Henzl, M.T.; Becker, D.F.; Tanner, J.J. Structure and characterization of a class $3 \mathrm{~B}$ proline utilization A: Ligand-induced dimerization and importance of the C-terminal domain for catalysis. J. Biol. Chem. 2017, 292, 9652-9665. [CrossRef] [PubMed]

3. Christgen, S.L.; Zhu, W.; Sanyal, N.; Bibi, B.; Tanner, J.J.; Becker, D.F. Discovery of the membrane binding domain in trifunctional proline utilization A. Biochemistry 2017, 56, 6292-6303. [CrossRef] [PubMed]

4. Arentson, B.W.; Luo, M.; Pemberton, T.A.; Tanner, J.J.; Becker, D.F. Kinetic and structural characterization of tunnel-perturbing mutants in Bradyrhizobium japonicum proline utilization A. Biochemistry 2014, 53, 5150-5161. [CrossRef] [PubMed]

5. Liu, L.; Becker, D.F.; Tanner, J.J. Structure, function, and mechanism of proline utilization A (PutA). Arch. Biochem. Biophys. 2017, 632, 142-157. [CrossRef] [PubMed]

6. Moxley, M.A.; Sanyal, N.; Krishnan, N.; Tanner, J.J.; Becker, D.F. Evidence for hysteretic substrate channeling in the proline dehydrogenase and $\Delta^{1}$-pyrroline-5-carboxylate dehydrogenase coupled reaction of proline utilization A (PutA). J. Biol. Chem. 2014, 289, 3639-3651. [CrossRef] [PubMed]

7. Singh, H.; Arentson, B.W.; Becker, D.F.; Tanner, J.J. Structures of the PutA peripheral membrane flavoenzyme reveal a dynamic substrate-channeling tunnel and the quinone-binding site. Proc. Natl. Acad. Sci. USA 2014, 111, 3389-3394. [CrossRef] [PubMed]

8. Srivastava, D.; Zhu, W.; Johnson, W.H., Jr.; Whitman, C.P.; Becker, D.F.; Tanner, J.J. The structure of the proline utilization A proline dehydrogenase domain inactivated by $N$-propargylglycine provides insight into conformational changes induced by substrate binding and flavin reduction. Biochemistry 2010, 49, 560-569. [CrossRef] [PubMed]

9. Surber, M.W.; Maloy, S. The PutA protein of Salmonella typhimurium catalyzes the two steps of proline degradation via a leaky channel. Arch. Biochem. Biophys. 1998, 354, 281-287. [CrossRef] [PubMed]

10. White, T.A.; Johnson, W.H., Jr.; Whitman, C.P.; Tanner, J.J. Structural basis for the inactivation of Thermus thermophilus proline dehydrogenase by $N$-propargylglycine. Biochemistry 2008, 47, 5573-5580. [CrossRef] [PubMed] 
11. Tanner, J.J.; Becker, D.F. PutA and proline metabolism. In Handbook of Flavoproteins. Volume 1-Oxidases, Dehydrogenases and Related Systems; Hille, R., Miller, S.M., Palfey, B., Eds.; De Gruyter: Berlin, Germany; Boston, MA, USA, 2012.

12. Tanner, J.J. Structural biology of proline catabolism. Amino Acids 2008, 35, 719-730. [CrossRef] [PubMed]

13. Lee, Y.H.; Nadaraia, S.; Gu, D.; Becker, D.F.; Tanner, J.J. Structure of the proline dehydrogenase domain of the multifunctional PutA flavoprotein. Nat. Struct. Biol. 2003, 10, 109-114. [CrossRef] [PubMed]

14. Zhang, M.; White, T.A.; Schuermann, J.P.; Baban, B.A.; Becker, D.F.; Tanner, J.J. Structures of the Escherichia coli PutA proline dehydrogenase domain in complex with competitive inhibitors. Biochemistry 2004, 43, 12539-12548. [CrossRef] [PubMed]

15. Tallarita, E.; Pollegioni, L.; Servi, S.; Molla, G. Expression in Escherichia coli of the catalytic domain of human proline oxidase. Protein Expr. Purif. 2012, 82, 345-351. [CrossRef] [PubMed]

16. Sanyal, N.; Arentson, B.W.; Luo, M.; Tanner, J.J.; Becker, D.F. First evidence for substrate channeling between proline catabolic enzymes: A validation of domain fusion analysis for predicting protein-protein interactions. J. Biol. Chem. 2015, 290, 2225-2234. [CrossRef] [PubMed]

17. Huijbers, M.M.E.; van Berkel, W.J.H. High yields of active Thermus thermophilus proline dehydrogenase are obtained using maltose-binding protein as a solubility tag. Biotechnol. J. 2015, 10, 395-403. [CrossRef] [PubMed]

18. Huijbers, M.M.E.; Martínez-Júlvez, M.; Westphal, A.H.; Delgado-Arciniega, E.; Medina, M.; van Berkel, W.J.H. Proline dehydrogenase from Thermus thermophilus does not discriminate between FAD and FMN as cofactor. Sci. Rep. 2017, 7. [CrossRef] [PubMed]

19. Huijbers, M.M.E.; van Berkel, W.J.H. A more polar N-terminal helix releases Thermus thermophilus proline dehydrogenase from self-association. J. Mol. Catal. B 2016, 134, 340-346. [CrossRef]

20. Eisenberg, D.; Schwarz, E.; Komaromy, M.; Wall, R. Analysis of membrane and surface protein sequences with the hydrophobic moment plot. J. Mol. Biol. 1984, 179, 125-142. [CrossRef]

21. Luo, M.; Arentson, B.W.; Srivastava, D.; Becker, D.F.; Tanner, J.J. Crystal structures and kinetics of monofunctional proline dehydrogenase provide insight into substrate recognition and conformational changes associated with flavin reduction and product release. Biochemistry 2012, 51, 10099-10108. [CrossRef] [PubMed]

22. Srivastava, D.; Schuermann, J.P.; White, T.A.; Krishnan, N.; Sanyal, N.; Hura, G.L.; Tan, A.; Henzl, M.T.; Becker, D.F.; Tanner, J.J. Crystal structure of the bifunctional proline utilization A flavoenzyme from Bradyrhizobium japonicum. Proc. Natl. Acad. Sci. USA 2010, 107, 2878-2883. [CrossRef] [PubMed]

23. Rowley, G.L.; Greenleaf, A.L.; Kenyon, G.L. On the specificity of creatine kinase. New glycocyamines and glycocyamine analogs related to creatine. J. Am. Chem. Soc. 1971, 93, 5542-5551. [CrossRef] [PubMed]

Sample Availability: Samples of the gene constructs reported here are available from the authors.

(C) 2018 by the authors. Licensee MDPI, Basel, Switzerland. This article is an open access article distributed under the terms and conditions of the Creative Commons Attribution (CC BY) license (http://creativecommons.org/licenses/by/4.0/). 